\title{
Comparative Analysis of Ant Colony and Particle Swarm Optimization Techniques
}

\author{
V.Selvi \\ Lecturer, \\ Department of Computer Science, \\ Nehru Memorial College, \\ Puthanampatti. Trichy.621005 \\ Tamilnadu. India.
}

\author{
Dr.R.Umarani \\ Associate professor, \\ Department of Computer Science, \\ Sri Saradha College for Women, \\ Salem. Tamilnadu \\ India.
}

\begin{abstract}
For a decade swarm Intelligence, an artificial intelligence discipline, is concerned with the design of intelligent multi-agent systems by taking inspiration from the collective behaviors of social insects and other animal societies. They are characterized by a decentralized way of working that mimics the behavior of the swarm. Swarm Intelligence is a successful paradigm for the algorithm with complex problems. This paper focuses on the comparative analysis of most successful methods of optimization techniques inspired by Swarm Intelligence (SI) : Ant Colony Optimization (ACO) and Particle Swarm Optimization (PSO). An elaborate comparative analysis is carried out to endow these algorithms with fitness sharing, aiming to investigate whether this improves performance which can be implemented in the evolutionary algorithms.
\end{abstract}

Keywords: Particle swarm optimization, Swarm intelligence, Ant Colony Optimization

\section{INTRODUCTION}

Swarm intelligence (SI), which is an artificial intelligence (AI) discipline, is concerned with the design of intelligent multi-agent systems by taking inspiration from the collective behavior of social insects such as ants, termites, bees, and wasps, as well as from other animal societies such as flocks of birds or schools of fish. Colonies of social insects have fascinated researchers for many years, and the mechanisms that govern their behavior remained unknown for a long time. Even though the single member of these colonies are non-sophisticated individuals, they are able to achieve complex tasks in cooperation. Coordinated colony behavior emerges from relatively simple actions or interactions between the colonies and the individual members. Many aspects of the collective activities of social insects are selforganized and work without a central control. Clustering means the act of partitioning an unlabeled dataset into groups of similar objects. Each group, called a cluster, consists of objects that are similar among themselves and dissimilar to objects of groups. In the past few decades, cluster analysis has played a central role in a variety of fields ranging from engineering (machine learning, artificial intelligence, pattern recognition, mechanical engineering, electrical engineering), computer sciences (web mining, spatial database analysis, textual document collection, image segmentation), life and medical 
sciences (genetics, biology, microbiology, paleontology, psychiatry, pathology), to earth sciences (geography, geology, remote sensing), social sciences (sociology, psychology, archeology, education), and economics (marketing, business) (Evangelou et al., 2001, Lillesand and Keifer, 1991, Rao, 1971, Duda and Hart, 1973, Fukunaga, 1990, Everitt, 1993). Swarm intelligence can be implemented in the field of clustering for obtaining approximately solutions to optimization problems in a reasonable amount of computation time. These are two important and recent methods of optimization such as ACO and PSO, which is implemented for this purpose.

The main properties of the collective behavior can be pointed out as follows and is summarized.

Homogeneity: Every bird in a flock has the same behavioral model. The flock moves without a leader, even though temporary leaders seem to appear.

Locality: its nearest flock mates only influence the motion of each bird. Vision is considered to be the most important senses for flock organization.

Collision Avoidance: avoid colliding with nearby flock mates.

Velocity Matching: attempt to match velocity with nearby flock mates.

Flock Centering: attempt to stay close to nearby flock mates

The ability of Particle Swarm Optimization (PSO), heuristic technique for search of optimal solutions based on the concept of swarm, to efficiently face classification of multiclass database instances. PSO reveals itself very effective in facing multivariable problems in which any variable takes on real values. It has roots in two methodologies. Its links to Artificial Life in general, and with bird flocks, fish schools and swarm theory in particular are very evident.
Nonetheless, PSO is also tied to Evolutionary Computation, namely to Genetic Algorithms (GA) and to Evolutionary Programming. The ACO and PSO can be analyzed for future enhancements such that new research could be focused to produce better solution by implementing the effectiveness and reducing the limitations of PSO. Plans to endow PSO with fitness sharing, aiming to investigate whether this helps in improving performance can be implemented in the evolutionary algorithms.

The rest of this paper is organized as follows: Section 2 describes Ant Colony Optimization. Section 3 describes Basic particle swarm optimization. Section 4 presents Proposed Methodology for particle swarm optimization. . Section 5 presents an Advantages and Disadvantages of ACO and PSO. Section 6 presents an Analysis of ACO and PSO. Section 7 presents an Applications of ACO and PSO. Section 8 concludes the paper and Future Work

\section{ANT COLONY OPTIMIZATION}

The Ant Colony Systems or the basic idea of a real ant system is illustrated in Figure 1. In the left picture, the ants move in a straight line to the food. The middle picture illustrates the situation soon after an obstacle is inserted between the nest and the food. To avoid the obstacle, initially each ant chooses to turn left or right at random. Let us assume that ants move at the same speed depositing pheromone in the trail uniformly. However, the ants that, by chance, choose to turn left will reach the food sooner, whereas the ants that go around the obstacle turning right will follow a longer path, and so will take longer time to circumvent the obstacle. As a result, pheromone accumulates faster in the shorter path around the obstacle. Since ants prefer to follow trails with larger amounts of pheromone, eventually all the ants converge to the shorter path around the obstacle, as shown in Figure 1 

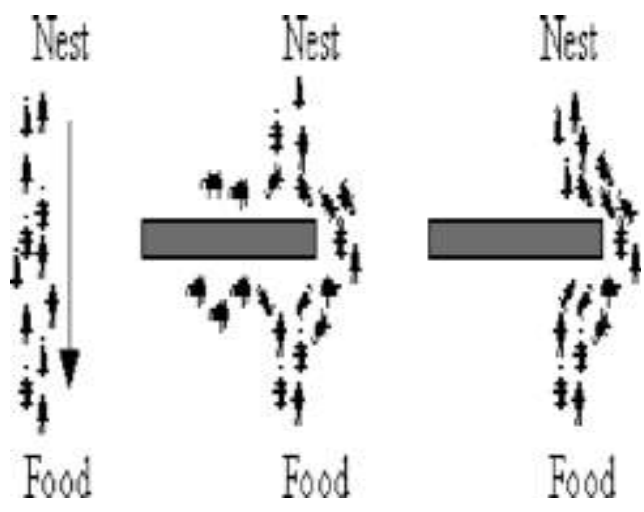

Fig. 1. Illustrating the behavior of real ant movements.

An artificial Ant Colony System (ACS) is an agent-based system, which simulates the natural behavior of ants and develops mechanisms of cooperation and learning. ACS was proposed by Dorigo et al. (Dorigo and Gambardella, 1997) as a new heuristic to solve combinatorial optimization problems. This new heuristic, called Ant Colony Optimization (ACO) has been found to be both robust and versatile in handling a wide range of combinatorial optimization problems.

The main idea of ACO is to model a problem as the search for a minimum cost path in a graph. Artificial ants as if walk on this graph, looking for cheaper paths. Each ant has a rather simple behavior capable of finding relatively costlier paths. Cheaper paths are found as the emergent result of the global cooperation among ants in the colony. The behavior of artificial ants is inspired from real ants: they lay pheromone trails (obviously in a mathematical form) on the graph edges and choose their path with respect to probabilities that depend on pheromone trails. These pheromone trails progressively decrease by evaporation. In addition, artificial ants have some extra features not seen in their counterpart in real ants. In particular, they live in a discrete world (a graph) and their moves consist of transitions from nodes to nodes.

The ACO differs from the classical ant system in the sense that here the pheromone trails are updated in two ways. Firstly, when ants construct a tour they locally change the amount of pheromone on the visited edges by a local updating role.

Secondly, after all the ants have built their individual tours, a global updating rule is applied to modify the pheromone level on the edges that belong to the best ant tour found so far.

\section{BASIC PARTICLE SWARM OPTIMIZATION}

Particle swarm optimization (PSO) is an algorithm modeled on swarm intelligence, that finds a solution to an optimization problem in a search space, or model and predict social behavior in the presence of objectives. The PSO is a stochastic, population-based computer algorithm modeled on swarm intelligence. Swarm intelligence is based on social-psychological principles and provides insights into social behavior, as well as contributing to engineering applications.

The particle swarm optimization algorithm was first described in 1995 by James Kennedy and Russell C. Eberhart. The particle swarm simulates this kind of social optimization. A problem is given, and some way to evaluate a proposed solution to it exists in the form of a fitness function. A communication structure or social network is also defined, assigning neighbors for each individual to interact with. Then a population of individuals defined as random guesses at the problem solutions is initialized. These individuals are candidate solutions. They are also known as the particles, hence the name particle swarm. An iterative process to improve these candidate solutions is set in motion. The particles iteratively evaluate 
the fitness of the candidate solutions and remember the location where they had their best success. The individual's best solution is called the particle best or the local best. Each particle makes this information available to their neighbors.

They are also able to see where their neighbors have had success. Movements through the search space are guided by these successes, with the population usually converging, by the end of a trial, on a problem solution better than that of non-swarm approach using the same methods. Each particle represents a candidate solution to the optimization problem. The position of a particle is influenced by the best position visited by itself i.e. its own experience and the position of the best particle in its neighborhood i.e. the experience of neighboring particles. When the neighborhood of a particle is the entire swarm, the best position in the neighborhood is referred to as the global best particle, and the resulting algorithm is referred to as the gbest PSO. When smaller neighborhoods are used, the algorithm is generally referred to as the lbest PSO. The performance of each particle is measured using a fitness function that varies depending on the optimization problem.

Each Particle in the swarm is represented by the following characteristics:

1. The current position of the particle

2. The current velocity of the particle

The particle swarm optimization which is one of the latest evolutionary optimization techniques conducts searches uses a population of particles.

Each particle corresponds to individual in evolutionary algorithm. Each particle has an updating position vector and updating velocity vector by moving through the problem space.

$\mathrm{V}_{\mathrm{i}}{ }^{\mathrm{k}+1}=w \mathrm{~V}_{\mathrm{i}}{ }^{\mathrm{k}}+\mathrm{c}_{1} \operatorname{rand}_{1}() \mathrm{x}\left(\right.$ pbest $\left._{\mathrm{i}}-\mathrm{s}_{\mathrm{i}}^{\mathrm{k}}\right)+\mathrm{c}_{2}$

$\operatorname{rand}_{2}() \times\left(\right.$ gbest- $\left._{\mathrm{i}}{ }^{\mathrm{k}}\right)$------- Eq (1)

$\mathrm{S}_{\mathrm{i}}{ }^{\mathrm{k}+1}=\mathrm{S}_{\mathrm{i}}{ }^{\mathrm{k}}+\mathrm{V}_{\mathrm{i}}{ }^{\mathrm{k}+1}$----- Eq (2)

Where,

$\mathrm{V}_{\mathrm{i}}{ }^{\mathrm{k}}$ is the velocity of $\mathrm{i}$ at iteration $\mathrm{k}, \mathrm{s}_{\mathrm{i}}{ }^{\mathrm{k}}$ is the current position of $\mathrm{i}$ at iteration $\mathrm{k} . \mathrm{c}_{1}$ and $\mathrm{c}_{2}$ are positive constants and rand $_{1}$ and $\operatorname{rand}_{2}$ are uniformly distributed random number in $[0,1]$. The velocity vector is range of [-Vmax, $\mathrm{Vmax}]$. In Velocity updating eq (1), eq (3) terms that creates new velocity are,

1. Inertia term, forces the particle to move in the same direction as before by adjusting the old velocity.

2. Cognitive term (Personal best), forces the particle to go back to the previous best position.

3. Social Learning term, forces the particle to move to the best previous position of its neighbors.

\section{ADVANTAGES AND DISADVANTAGES}

\subsection{An Analysis on the Advantages and Disadvantages of the Basic Particle Swarm Optimization Algorithm. Advantages of the basic particle swarm
optimization algorithm:}

(1)PSO is based on the intelligence. It can be applied into both scientific research and engineering use.

(2)PSO have no overlapping and mutation calculation. The search can be carried out by the speed of the particle. During the development of several generations, only the most optimist particle can transmit information onto the other particles, and the speed of the researching is very fast.

(3)The calculation in PSO is very simple. Compared with the other developing calculations, it occupies the bigger optimization ability and it can be completed easily.

(4) PSO adopts the real number code, and it is decided directly by the solution. The number of the dimension is equal to the constant of the solution.

Disadvantages of the basic particle swarm optimization algorithm:

(1)The method easily suffers from the partial 
optimism, which causes the less exact at the regulation of its speed and the direction.

(2)The method cannot work out the problems of scattering and optimization.

(3)The method cannot work out the problems of non-coordinate system, such as the solution to the energy field and the moving rules of the particles in the energy field

\subsection{An Analysis on the Advantages and Disadvantages of the Ant Colony Optimization.}

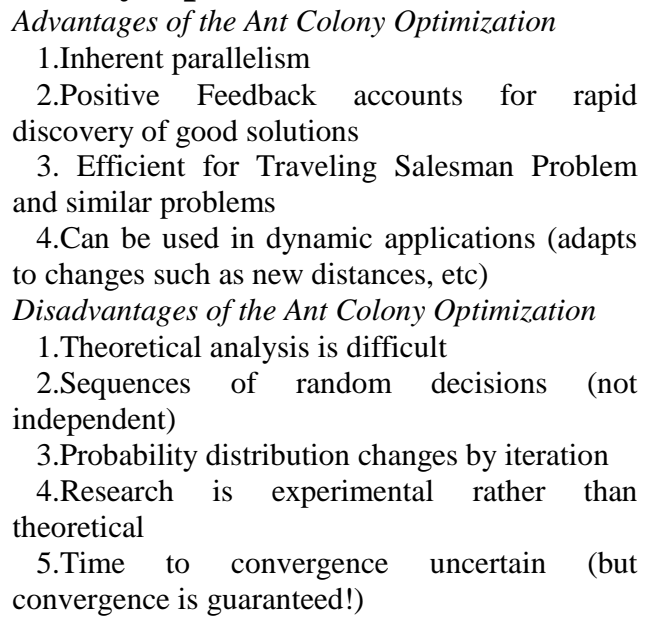

\section{ANALYSIS OF ACO AND PSO}

The ACO is inspired by the aging behaviors of ant colonies. At the core of this behaviors the indirect communication between the ants enables them to find short paths between their nest and food sources. This characteristic of real ant colonies is exploited in ACO algorithm to solve, discrete optimization problems. The PSO technique modeled on the social behaviors observed is animals or insects, PCO has gained increasing popularity among researches and practitioners as a robust and efficient technique for solving difficult robust and population nbased stochastic optimization problems.
Both the ACO and PSO algorithm are the data clustering algorithms by implementing swarm behavior. Whereas the ACO is more applicable for problems where source and destination are predefined and specific. At the same time PSO is a clustering algorithm in the areas of mutliobjective, dynamic optimization and constraint handling. The ACO is more applicable for problems that requires crisps results and PSO is applicable for problems that are fuzzy is nature. All these characteristic of the ACO and PSO are implicitly evident in the following applications.

\section{APPLICATIONS OF ACO AND PSO}

Ant colony optimization algorithms have been applied to many combinatorial optimization problems, ranging from quadratic assignment to fold protein or routing vehicles and a lot of derived methods have been adapted to dynamic problems in real variables, stochastic problems, multi-targets and parallel implementations. It has also been used to produce near-optimal solutions to the travelling salesman problem. They have an advantage over simulated annealing and genetic algorithm approaches of similar problems when the graph may change dynamically; the ant colony algorithm can be run continuously and adapt to changes in real time. This is of interest in network routing and urban transportation systems.

The first practical application of PSO was in the field of neural network training and was reported together with the algorithm itself (Kennedy and Eberhart 1995). Many more areas of application have been explored ever since, including telecommunications, control, data mining, design, combinatorial optimization, power systems, signal processing, and many others. To date, there are hundreds of publications reporting applications of particle swarm optimization algorithms. For a review, see (Poli 2008). Although PSO has been used mainly to solve unconstrained, single-objective optimization 
problems, PSO algorithms have been developed to solve constrained problems, multi-objective optimization problems, problems with dynamically changing landscapes, and to find multiple solutions.

\section{CONCLUSIONS AND FUTURE WORK}

The ACO and PSO can be analyzed for future enhancement such that new research could be focused to produce better solution by improving the effectiveness and reducing the limitations. More possibilities for dynamically determining the best destination through ACO can be evolved and a plan to endow PSO with fitness sharing aiming to investigate whether this helps in improving performance. In future the velocity of each individual must be updated by taking the best element found in all iterations rather than that of the current iteration only.

\section{REFERENCES}

[1]. Ajith Abraham,Swagatam Das,Sandip Roy, "Swarm Intelligence Algorithms for Data Cluctering.
[2]. E.Corchado, PSO and ACO in Optimization Problems , Publishers : Springer - Verlag, pp. 1390 - 1398, 2006.

[3]. Tsang W and Kwongs S, Ant Colony Clustering and Feature Extraction for Anomaly Intrusion Detection, in Swarm intelligence in Data Mining, Abraham A,(2006).

[4]. Macro Dorigo, The Ant System: Optimization by a colony of cooperating agents.

[5]. Christian Blum and Xiaodong Li, Swarm Intelligence in Optimization.

[6]. P.Mathiyalagan, Grid scheduling Using Enhanced PSO Algorithm International Journal on Computer Science and Engineering,Vol. 02, No. $02,2010,140-145$

[7]. R. S. Parpinelli, H. S. Lopes, and A.A. Freiatas. Data mining with an ant colony optimization algorithm. IEEE Transactions on evolutionary computation, 6(4):321-332, 2002.

[8]. De Falco, A. Della Cioppa, and E. Tarntino, "Evalution of Particle Swarm Optimization Effectiveness in Classification.

[9].Qinghai Bai,Analysis of Particle Swarm Optimization Algorithm Computer and Information Science Vol:3 ,No.1,February 2010. 